\title{
Intravascular catheter bloodstream infections: an effective and sustained hospital-wide prevention program over 8 years
}

\author{
Peter J Collignon, Dianne E Dreimanis, Wendy D Beckingham, Jan L Roberts and Anne Gardner
}

$\mathrm{M}$ any commonly occurring health care-associated infections are lifethreatening, especially bloodstream infections (BSIs). ${ }^{1,2}$ Intravascular (IV) catheters are the most frequent cause of BSIs, most of which are preventable. . $^{3-8}$

In the United States, BSIs are now the eighth leading cause of death, ${ }^{1}$ and 250000 episodes associated with central venous catheters (CVCs) are estimated to occur there annually. ${ }^{3}$ In Australia in the early 1990s, about $2 \%$ of all CVCs and $0.04 \%$ of peripheral venous catheters were associated with BSIs, resulting in over 3000 catheter-related BSI episodes per year and an average rate of 1.6 per 1000 admissions in Australian teaching hospitals. ${ }^{4}$ More recently in England, this rate was 2.3 per 1000 admissions in teaching hospitals and 0.9 per 1000 admissions in non-teaching hospitals. ${ }^{7}$ The reported mortality associated with BSIs is variable, ${ }^{3}$ but in the US even a low rate of attributable mortality of $10 \%$ translates to 25000 deaths per year.

Ongoing surveillance of BSIs associated with IV catheters is patchy. Available "whole of hospital" data show that IV catheters account for $30 \%$ or more of BSIs in Australia, England and the US. ${ }^{5-7,9}$ Data collection and surveillance of BSIs has been recommended, but primarily for intensive care units (ICUs), ${ }^{10,11}$ even though most CVCs are used outside ICUs. ${ }^{12}$

To prevent the occurrence of BSIs and improve management practices, we implemented a hospital-wide surveillance and intervention program.

\section{METHODS}

Canberra Hospital is a 500-bed tertiary referral hospital offering most medical and surgical services for a population of over 550000 . In 1990, the hospital's rate of IV catheterrelated BSIs was 1.7 per 1000 discharges. ${ }^{4}$

We used a quality assurance grant to employ a full-time nurse and a part-time data entry clerk (0.2 full-time equivalent [FTE]), and to purchase computer hardware and software. A part-time nurse (0.6 FTE) was employed once the program was established (total recurrent costs per annum of about A $\$ 60000$ ). Our surveillance study ran for 8 years, from 1998 to 2005

\section{ABSTRACT}

Objective: To evaluate a hospital-wide surveillance and intervention program introduced to reduce the incidence of bloodstream infections (BSIs) caused by intravascular (IV) catheters.

Design, setting and participants: Prospective surveillance of all inpatients and outpatient attendees with positive blood cultures (both hospital-onset and community-onset) at a 500-bed tertiary referral hospital from 1998 to 2005.

Interventions: Prompt review of all positive blood cultures with identification of BSIs due to IV catheters and associated preventable factors; weekly team meetings and regular reports to clinical areas, with assistance to implement targeted interventions.

Main outcome measure: Number of BSI episodes due to IV catheters per year. Results: There were $491 \mathrm{BSI}$ episodes due to IV catheters, mainly central venous catheters. Episodes per year fell from 110 in 1998 to 48 in 2005 (from 32\% of all BSI episodes to $14 \%$; a > 50\% reduction). From 1998 to 2005, the rate per 1000 discharges fell from 2.3 to 0.9 ( $P$ for trend $<0.0005$ ) and the rate per 1000 patient-days fell from 0.6 to 0.3 ( $P$ for trend $<0.0005$ ).

Conclusions: Our program was associated with a profound drop in the number of IV catheter-related BSIs per year. Active surveillance and intervention programs can lead to substantial and sustained reductions in these common life-threatening infections.

MJA 2007; 187: 551-554

The project nurse visited the microbiology laboratory daily to review all BSI episodes (both hospital- and community-onset) after receiving notification of positive blood cultures. The nurse also visited wards to obtain episode details from the patient, the caring medical and nursing staff, and medical records. Episodes were categorised using national definitions. ${ }^{13}$ IV catheter-related BSIs and primary health care-associated bacteraemias were categorised using US Centers for Disease Control and Prevention definitions. ${ }^{3}$ At Day 7 or earlier if discharged, the patient's outcome (alive or not) and the contribution of sepsis to mortality or ongoing morbidity were assessed.

A multidisciplinary team reviewed the clinical details of all new positive blood culture results at a 30-minute weekly meeting. Particular attention was paid to any episode potentially associated with IV catheter sepsis or preventable causes.

Data were obtained from the hospital's financial and activity reports for occupied bed-days (here referred to as "patient-days") and separations ("discharges") and included day-only patients. Trends in the annual rate of BSIs per patient-days and discharges were estimated by aggregating data for BSIs, patient-days and discharges for each calendar year. Rates were modelled in Stata (StataCorp, College Station, Tex, USA) using log linear regression of the number of BSIs, with the logarithm of the number of patient-days or discharges as the offset term. Year was fitted as a linear term to assess whether there was a trend, but without modelling the trend itself. Significance of the regression term was assessed using a likelihood ratio test at the 5\% level. Rate ratios for 2005 versus 1998 were calculated using the fitted values for the number of BSIs.

This study was a quality assurance program but was also approved by the ACT Health Human Research Ethics Committee.

\section{RESULTS}

Over the 8-year period, there were 491 BSI episodes caused by IV catheters, with a greater than $50 \%$ reduction during the study, from 110 episodes in 1998 to 48 in 2005 (Box 1, Box 2). From 1998 to 2005, the rate of IV catheter-related episodes fell from 2.3 to 0.9 per 1000 discharges ( $P$ for trend $<0.0005$ ), and from 0.6 to 0.3 per 1000 patient-days ( $P$ for trend $<0.0005)$. The rate ratio was 0.35 (95\% CI, 0.26-0.46) for discharges and $0.36(95 \% \mathrm{CI}, 0.27-0.48)$ for patient-days. 


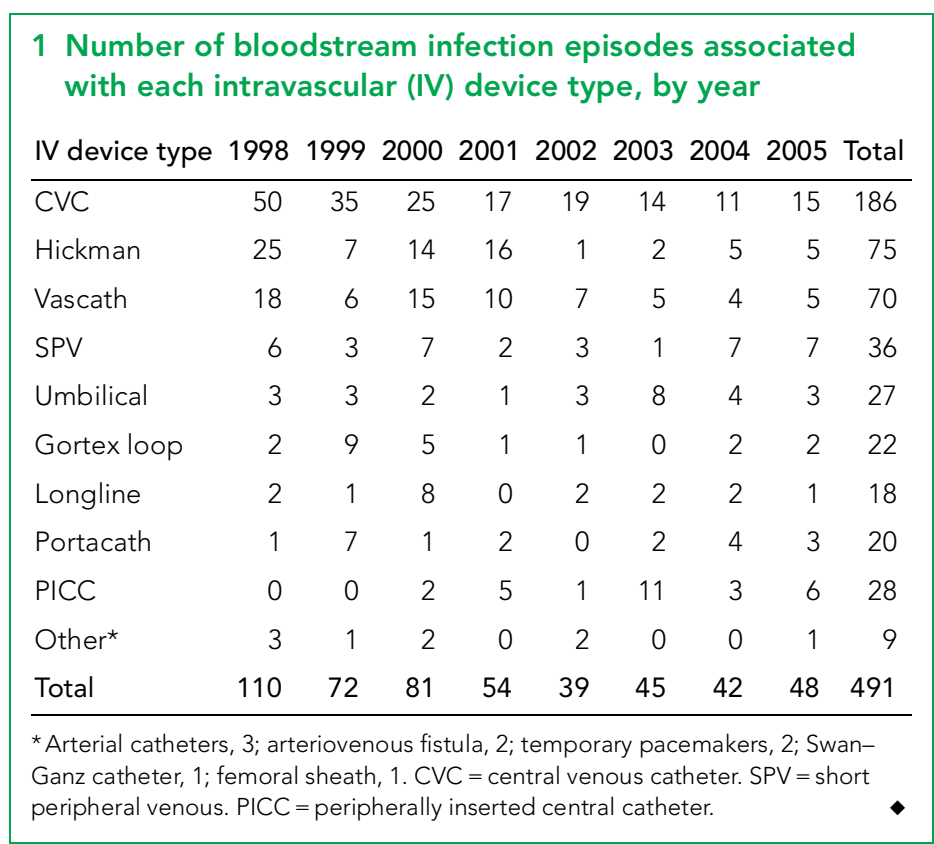

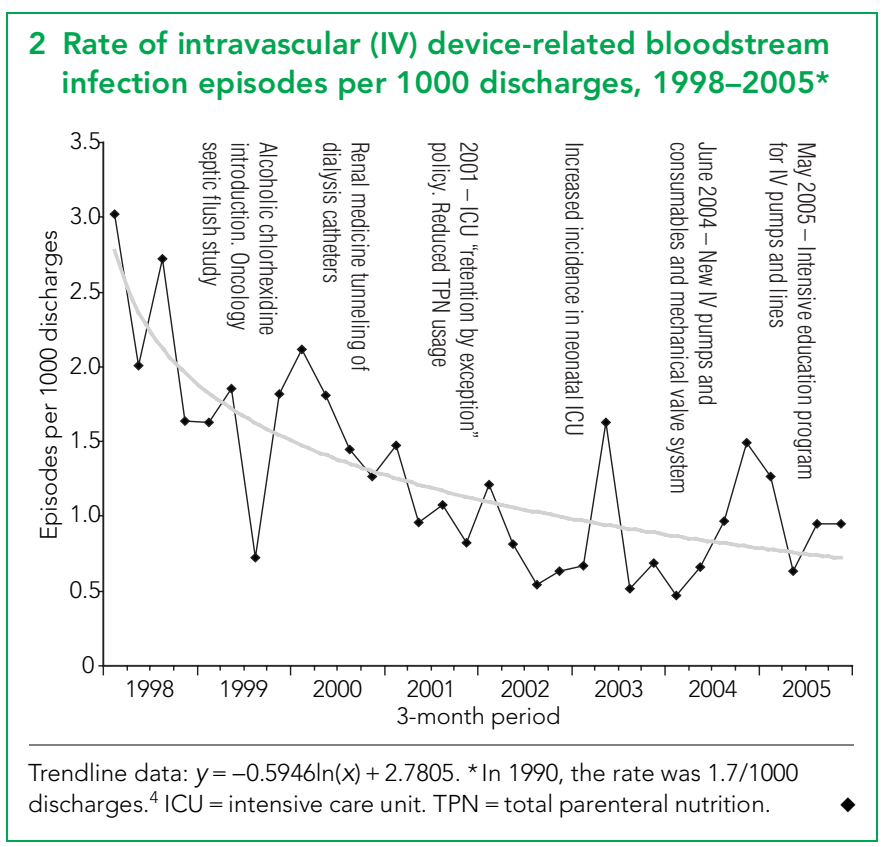

Although the number of episodes in each 3-month period fluctuated, there was an overall downward trend from the beginning to the end of the project (Box 2). Episodes of IV catheter sepsis resulted in $4 \%$ mortality (20/491 episodes) by Day 7. A subgroup of 44 patients with IV catheter sepsis in 20002001 was followed for 6 months, and had a cumulative mortality of $4 \%$ at Day $7,12 \%$ at 1 month, and 39\% at 6 months.

CVCs were the most common IV catheters associated with BSIs (Box 1). However, all catheter types were implicated, including short peripheral catheters and peripherally inserted central catheters (PICC lines).

IV catheter sepsis occurred in all areas of the hospital (Box 3), but the numbers in each unit varied from year to year. In 2004, there were 1238 CVCs, 617 PICC lines and 27683 peripheral catheters purchased, with BSI rates of 8.8 per 1000 CVC catheters (0.9\%), 4.9 per 1000 PICC lines $(0.5 \%)$, and 0.25 per 1000 peripheral catheters $(0.03 \%)$.

Staphylococcus aureus (methicillin-sensitive and methicillin-resistant) was the most common organism involved in IV sepsis, followed by coagulase-negative staphylococci and Candida spp. (Box 4). Comparing 1998-2001 with 2002-2005, the largest percentage falls were with $S$. aureus, coagulase-negative staphylococci, and gram-negative rods.

In 1998, IV catheters were the most common primary site for sepsis when all BSI episodes from any cause were examined, accounting for 110 of the 339 significant episodes (32\%) (Box 5). After 2002, IV catheters were no longer the commonest cause of
BSIs; by 2005 they had decreased to be the third most common primary site (48/341 episodes; 14\%) after gastrointestinal tract and genitourinary tract episodes. Primary health care-related episodes (ie, all IV and all "unknown" primary site BSIs) dropped from 128 episodes in 1998 to 87 in 2005 (Box 5). In 2005, this was a rate of 1.7 per 1000 discharges; down 38\% from 1998.

\section{DISCUSSION}

The decline in IV catheter-related BSIs was associated with multiple interventions. Individual units instigated many of these, usually in consultation with the infection control service after receiving feedback and advice about their rates and numbers of IV-related BSIs (Box 6). We believe our surveillance program was the catalyst for many of these interventions, which may otherwise have been introduced later or not at all.

The project nurse's ward visits to discuss each BSI episode with the medical and nursing staff provided prompt feedback. This interaction and the collection and feedback of other data allowed the units to "buy in" to the problem. We believe the combined interaction of all these program elements resulted in the dramatic reduction in sepsis observed; a point also made by others. ${ }^{10}$ Reliable and regular reporting of results also sustained enthusiasm for ongoing vigilance.

Passive surveillance based on medical and laboratory records alone is unlikely to have achieved the same positive outcomes. With its associated time lag, passive surveillance is

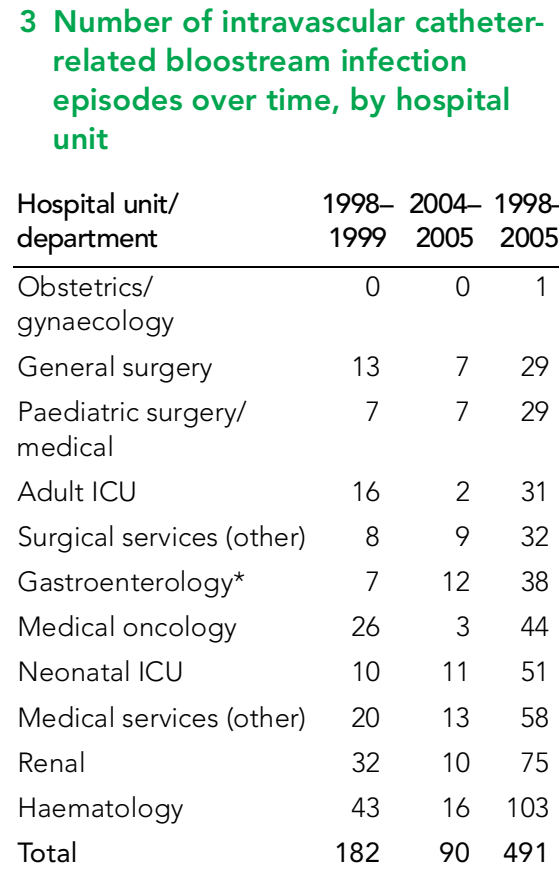

* Frequently associated with total parenteral nutrition. ICU = intensive care unit.

also likely to have been much less accurate in identifying the primary sites for sepsis or patient outcome. Studies in ICUs in the US, Germany, Brazil, Australia and Switzerland have shown that active surveillance and interventions can substantially reduce the number of BSIs caused by IV catheters. $8,10,11,14-18$ Our study confirms the findings of Meier et $\mathrm{al}^{19}$ that this can also be done for the whole hospital. 


\begin{tabular}{|c|c|c|c|c|c|c|c|c|c|}
\hline Microorganism* & 1998 & 1999 & 2000 & 2001 & 2002 & 2003 & 2004 & 2005 & Total (\%) \\
\hline Staphylococcus aureus & 26 & 30 & 29 & 20 & 20 & 14 & 13 & 13 & $165(31 \%)$ \\
\hline Methicillin-sensitive & 24 & 25 & 19 & 16 & 14 & 11 & 11 & 10 & $130(25 \%)$ \\
\hline Methicillin-resistant & 2 & 5 & 10 & 4 & 6 & 3 & 2 & 3 & $35(7 \%)$ \\
\hline $\begin{array}{l}\text { Coagulase-negative } \\
\text { staphylococci }\end{array}$ & 42 & 14 & 24 & 9 & 8 & 22 & 8 & 11 & $138(26 \%)$ \\
\hline Candida spp. & 8 & 1 & 4 & 4 & 1 & 0 & 8 & 8 & $34(6 \%)$ \\
\hline Klebsiella spp. & 7 & 3 & 6 & 7 & 1 & 3 & 1 & 3 & $31(6 \%)$ \\
\hline $\begin{array}{l}\text { Stenotrophomonas } \\
\text { maltophilia }\end{array}$ & 6 & 8 & 2 & 3 & 0 & 1 & 2 & 1 & $23(4 \%)$ \\
\hline Enterobacter spp. & 4 & 2 & 5 & 4 & 1 & 1 & 2 & 0 & 19 (4\%) \\
\hline Escherichia coli & 2 & 2 & 1 & 1 & 2 & 0 & 4 & 0 & $12(2 \%)$ \\
\hline Pseudomonas spp. & 4 & 3 & 3 & 3 & 2 & 0 & 2 & 4 & $21(4 \%)$ \\
\hline Other ${ }^{\dagger}$ & 17 & 14 & 11 & 6 & 7 & 7 & 10 & 12 & $84(16 \%)$ \\
\hline Total & 116 & 77 & 85 & 57 & 42 & 48 & 50 & 52 & $527(100 \%)$ \\
\hline
\end{tabular}

* In 31 episodes, there was more than one significant organism isolated. †The most common "other" isolates were: Viridans streptococci, 12; Acinetobacter spp., 10; Proteus spp., 10; Enterococcus spp., 10; Serratia spp., 8; and Corynebacterium jeikeium, 4

5 Rates of intravascular (IV) catheter sepsis and hospital admission details, by year

\begin{tabular}{|c|c|c|c|c|c|c|c|c|}
\hline & 1998 & 1999 & 2000 & 2001 & 2002 & 2003 & 2004 & 2005 \\
\hline Total significant episodes* & 339 & 308 & 318 & 289 & 272 & 316 & 354 & 341 \\
\hline Total indeterminate episodes & 35 & 37 & 37 & 36 & 31 & 32 & 25 & 35 \\
\hline Total contaminant episodes & 245 & 200 & 197 & 197 & 217 & 210 & 235 & 266 \\
\hline Total positive blood cultures & 619 & 545 & 552 & 522 & 520 & 558 & 614 & 642 \\
\hline IV catheter-related episodes & 110 & 72 & 81 & 54 & 39 & 45 & 42 & 48 \\
\hline $\begin{array}{l}\text { Health care-related primary } \\
\text { bacteraemia }\end{array}$ & 128 & 111 & 106 & 92 & 73 & 75 & 71 & 87 \\
\hline $\begin{array}{l}\text { Total significant episodes } \\
\text { excluding IV catheters }\end{array}$ & 228 & 235 & 239 & 234 & 232 & 271 & 312 & 291 \\
\hline Discharges & 46656 & 47454 & 49090 & 49606 & 49400 & 50805 & 48655 & 51122 \\
\hline \multicolumn{9}{|l|}{ Rate per 1000 discharges of: } \\
\hline Total significant episodes & 7.2 & 6.5 & 6.5 & 5.8 & 5.5 & 6.2 & 7.2 & 6.6 \\
\hline $\begin{array}{l}\text { Total significant episodes } \\
\text { excluding IV catheters }\end{array}$ & 4.9 & 5.0 & 4.9 & 4.7 & 4.7 & 5.3 & 6.4 & 5.7 \\
\hline IV-related episodes $^{\dagger}$ & 2.3 & 1.5 & 1.7 & 1.1 & 0.8 & 0.9 & 0.9 & 0.9 \\
\hline Patient-days $s^{\ddagger}$ & 171455 & 164027 & 166537 & 170285 & 170661 & 173833 & 171173 & 177239 \\
\hline \multicolumn{9}{|c|}{ Rate per 1000 patient-days of: } \\
\hline Total significant episodes & 2.0 & 1.9 & 1.9 & 1.7 & 1.6 & 1.8 & 2.1 & 1.9 \\
\hline $\begin{array}{l}\text { Total significant episodes } \\
\text { excluding IV catheters }\end{array}$ & 1.3 & 1.4 & 1.4 & 1.4 & 1.4 & 1.6 & 1.8 & 1.6 \\
\hline IV-related episodes & 0.6 & 0.4 & 0.5 & 0.3 & 0.2 & 0.3 & 0.2 & 0.3 \\
\hline
\end{tabular}

There are now at least 60 fewer BSI episodes occurring per year in our hospital than when the program started. This is likely to represent a saving of six or more lives per year (assuming at least 10\% attributable mortality). Each IV catheter-related BSI episode also has significant financial costs. Measuring these is beyond the scope of this study, but a
South Australian study found that each case of $S$. aureus bacteraemia may be associated with additional health care costs of about A $\$ 20000 .{ }^{20}$ In the US, the attributable cost of each CVC-associated BSI episode (from all organisms) is estimated at US\$34508$\$ 56000$. $^{3}$ Using the most conservative of these estimates, if 60 bacteraemia cases are prevented each year, and at least 15 of these are due to $S$. aureus, then annual hospital cost savings will be at least $A \$ 300000$. However, a more realistic estimate is over a million dollars per year. This is offset by the net additional cost of the program (about A $\$ 60000$ per year).

Our rate per 1000 patient-days for primary nosocomial BSIs fell 34\% from 0.8 in 1998 to 0.5 in 2005. A study in a 200-bed hospital in Iowa found a similar decrease in primary BSIs (35\%) after the introduction of an 11-member IV team (from 1.1 to 0.7 per 1000 patient-days). ${ }^{19}$ We were able to sustain a large reduction in IV-related sepsis, over a longer study period and at a much lower cost than with a full IV team.

Our data show the value of an ongoing surveillance program. On several occasions during the study we saw rising rates and then "peaks" in sepsis (Box 2). One was in 2003 in the neonatal ICU and prompted a complete review of their IV catheter-related procedures. We never determined the cause of this increase, but believe its early detection, in conjunction with the staff reviewing protocols and enacting changes, resulted in its resolution. The same benefit was noted with another peak in 2004 after the introduction of a new mechanical valve needleless IV system and new mechanical pumps. Similar increases of IV sepsis with use of these systems have been reported in the US. ${ }^{21}$ After an extensive education program at our hospital, IV sepsis rates returned to lower levels.

Programs that use only denominators such as "catheter line-days" and focus on ICUs are inappropriate. Whole-of-hospital programs should be implemented to capture all BSI episodes, with denominators that are easier to collect (eg, episodes per 1000 discharges or patient-days). ${ }^{7,8}$ Sepsis rates expressed only as catheter line-days do not acknowledge timely removal of lines and thus removal of risk, ${ }^{14}$ and may lead to continued overuse of these catheters and help to hide the numbers of episodes that occur.

The initial rate of IV sepsis in our hospital was reasonably low by most Australian ${ }^{4}$ and international comparisons. ${ }^{3}$ Our ICU rates were lower than those reported for comparable units in New South Wales (1.8 v 3.7 per 1000 line-days $)^{14,22}$ and than the average ICU rate in the US (5.3 per 1000 line-days for CVCs). ${ }^{3}$ Our study shows that a surveillance and intervention program can decrease BSI episodes even in hospitals with relatively low rates of sepsis. We believe that over $75 \%$ of episodes that still occur are due to preventable factors. Thus, the baseline number of "unavoidable" BSIs due to IV catheters should 
6 Interventions for intravascular (IV) catheter-related bloodstream infections (BSIs)

\section{Intervention}

Prospective surveillance of BSIs

Subcutaneous tunnelling of Vascath catheters

Central venous catheter (CVC) "retention by exception"*

Prevention of septic flush by correct use of alcohol antiseptics

Monitoring of peripheral IV policy compliance

Patient information pamphlet for CVC care

Introduction of alcoholic chlorhexidine skin prep for IV insertions

Reduction in the use of total parenteral nutrition

Notification of each IV catheter-related BSI to specialist in charge Hospital-wide, medical of patient by letter outlining current hospital policies and pointing officer

out potential breaches in the episode

Extensive education after introduction of new infusion pumps and Hospital-wide, general consumables practitioners

Dissemination of BSI project information

Hospital-wide, GPs, media

* The CVC was routinely removed when the patient left the ICU unless a specific and appropriate reason could be identified and documented to justify its continued retention (see references ${ }^{14,15}$ for more details).

be only about 15 episodes per year, or 0.2 per 1000 discharges.

A similar approach can be put into place in most hospitals. Our surveillance and intervention program is relatively inexpensive and likely to be extremely cost-effective. Programs such as this should result in a major reduction in IV catheter-related sepsis.

\section{ACKNOWLEDGEMENTS}

Funding for this project was through a Quality Assurance Grant from ACT Health that covered the salary of Jan Roberts. We thank Dr Margaret Staples, Biostatistician, Monash Department of Clinical Epidemiology and Cabrini Hospital Department of Epidemiology and Preventive Medicine, for her statistical advice. We also thank all those in the microbiology unit, others who worked part-time or as relief in the infection control service, and all those in other units who collected data or were involved in implementing changes in our hospital.

\section{COMPETING INTERESTS}

Travel assistance was received from Becton Dickinson Pty Ltd (supplier of IV infusion equipment) for Di Dreimanis to attend an international conference in July 2006, and for Jan Roberts and Wendy Beckingham to attend an interstate meeting in 2005.

\section{AUTHOR DETAILS}

Peter J Collignon, FASM, FRCPA, FRACP, Director, Infectious Diseases Unit and Microbiology Department, ${ }^{1}$ and Professor ${ }^{2}$ Dianne E Dreimanis, BHSc(Nursing), MN, Infection Control Practitioner, Infection Control ${ }^{1}$ Wendy D Beckingham, RN, BHSc(Nursing), Infection Control Practitioner, Infection Control ${ }^{1}$ Jan L Roberts, RN, BHSc(Nursing), Infection Control Practitioner, Infection Control ${ }^{1}$

Anne Gardner, BA, PhD, Professor ${ }^{3}$ (Previously Nursing Research ${ }^{1}$ )
1 Canberra Hospital, Canberra, ACT.

2 Canberra Clinical School, Australian National University, Canberra, ACT.

3 James Cook University and Townsville Health Service District, Townsville, QLD.

Correspondence: Peter.Collignon@act.gov.au

\section{REFERENCES}

1 Wenzel RP, Edmond MB. The impact of hospitalacquired bloodstream infections. Emerg Infect Dis 2001; 7: 174-177.

2 Martin GS, Mannino DM, Eaton S, Moss M. The epidemiology of sepsis in the United States from 1979 through 2000. N Engl J Med 2003; 348: 1546-1554.

3 O'Grady NP, Alexander M, Dellinger EP, et al. Guidelines for the prevention of intravascular catheter-related infections. Centers for Disease Control and Prevention. MMWR Recomm Rep 2002; 51(RR-10): 1-29. http://www.cdc.gov/ mmwr/PDF/rr/rr5110.pdf (accessed Apr 2006).

4 Collignon PJ. Intravascular catheter associated sepsis: a common problem. The Australian Study on Intravascular Catheter Associated Sepsis. Med J Aust 1994; 161: 374-378.

5 McGregor AR, Collignon PJ. Bacteraemia and fungaemia in an Australian general hospital associations and outcomes. Med J Aust 1993; 158: 671-674.

6 Weinstein MP, Towns ML, Quartey SM, et al. The clinical significance of positive blood cultures in the 1990s: a prospective comprehensive evaluation of the microbiology, epidemiology, and outcome of bacteremia and fungemia in adults. Clin Infect Dis 1997; 24: 584-602.

7 Coello R, Charlett A, Ward V, et al. Devicerelated sources of bacteraemia in English hospitals - opportunities for the prevention of hospital-acquired bacteraemia. J Hosp Infect 2003; 53: 46-57.

8 Eggimann $\mathrm{P}$, Harbarth S, Constantin MN, et al. Impact of a prevention strategy targeted at vascular-access care on incidence of infections acquired in intensive care. Lancet 2000; 355: 1864-1868.
9 Friedman ND, Kaye KS, Stout JE, et al. Health care-associated bloodstream infections in adults: a reason to change the accepted definition of community-acquired infections. Ann Intern Med 2002; 137: 791-797.

10 Gaynes R, Richards C, Edwards J, et al. Feeding back surveillance data to prevent hospitalacquired infections. Emerg Infect Dis 2001; 7: 295-298.

11 Centers for Disease Control and Prevention (CDC). Monitoring hospital-acquired infections to promote patient safety - United States 1990-1999. MMWR Morb Mortal Wkly Rep 2000; 49: 149-53. http://www.cdc.gov/mmwr/preview/ $\mathrm{mmwrhtml} / \mathrm{mm} 4908 \mathrm{a} 1$. htm (accessed Apr 2006).

12 Trick WE, Vernon MO, Welbel SF, et al. Unnecessary use of central venous catheters: the need to look outside the intensive care unit. Infect Control Hosp Epidemiol 2004; 25: 266-268.

13 Australian Infection Control Association Expert Working Group. Blood stream infection (BSI) definition. Approved by Health Care Associated Infection Advisory Committee. Canberra: Australian Council for Safety and Quality in Health Care, Sep 2004.

14 Gowardman JR, Kelaher C, Whiting J, Collignon PJ. Impact of a formal removal policy for central venous catheters on duration of catheterisation [letter]. Med J Aust 2005; 182: 249-250.

15 Lobo RD, Levin AS, Gomes LM, et al. Impact of an educational program and policy changes on decreasing catheter-associated bloodstream infections in a medical intensive care unit in Brazil. Am J Infect Control 2005; 33: 83-87.

16 Berenholtz SM, Pronovost PJ, Lipsett PA, et al. Eliminating catheter-related bloodstream infections in the intensive care unit. Crit Care Med 2004; 32: 2014-2020.

17 Kritchevsky SB, Braun BI, Wong ES, et al. Impact of hospital care on incidence of bloodstream infection: the evaluation of processes and indicators in infection control study. Emerg Infect Dis 2001; 7: 193-196.

18 Zuschneid I, Schwab F, Geffers C, et al. Reducing central venous catheter-associated primary bloodstream infections in intensive care units is possible: data from the German nosocomial infection surveillance system. Infect Control Hosp Epidemiol 2003; 24: 501-505.

19 Meier PA, Fredrickson M, Catney M, Nettleman $M D$. Impact of a dedicated intravenous therapy team on nosocomial bloodstream infection rates. Am J Infect Control 1998; 26: 388-392.

20 Australian Infection Control Association Expert Working Group. National surveillance of health care associated infection in Australia. A report to the Commonwealth Department of Health and Aged Care. April 2001. http:// www.health.gov.au/internet/wcms/publishing.nsf/Content/health-pubhlth-strateg-jetacararchive-index.htm/\$FILE/scope.pdf (accessed Apr 2006).

21 Maragakis LL, Bradley KL, Song $\mathrm{X}$, et al Increased catheter-related bloodstream infection rates after the introduction of a new mechanical valve intravenous access port. Infect Control Hosp Epidemiol 2006; 27: 67-70.

22 McLaws ML, Berry G. Nonuniform risk of bloodstream infection with increasing central venous catheter-days. Infect Control Hosp Epidemiol 2005; 26: 715-719.

(Received 6 Feb 2007, accepted 20 Aug 2007) 